\title{
On Sums of Products of Horadam Numbers
}

\author{
ZVONKO ČERIN \\ Kopernikova 7, 10010 Zagreb, Croatia, Europe \\ e-mail : cerin@math.hr
}

ABSTRACT. In this paper we give formulae for sums of products of two Horadam type generalized Fibonacci numbers with the same recurrence equation and with possibly different initial conditions. Analogous improved alternating sums are also studied as well as various derived sums when terms are multiplied either by binomial coefficients or by members of the sequence of natural numbers. These formulae are related to the recent work of Belbachir and Bencherif, Čerin and Čerin and Gianella.

\section{Introduction}

The generalized Fibonacci sequence $\left\{w_{n}\right\}=\left\{w_{n}\left(a_{0}, b_{0} ; p, q\right)\right\}$ is defined by

$$
w_{0}=a_{0}, \quad w_{1}=b_{0}, \quad w_{n}=p w_{n-1}-q w_{n-2} \quad(n \geq 2),
$$

where $a_{0}, b_{0}, p$ and $q$ are arbitrary complex numbers, with $q \neq 0$. The numbers $w_{n}$ have been studied by Horadam (see, e.g. [10]). A useful and interesting special cases are $\left\{U_{n}\right\}=\left\{w_{n}(0,1 ; p, q)\right\}$ and $\left\{V_{n}\right\}=\left\{w_{n}(2, p ; p, q)\right\}$ that were investigated by Lucas [11].

For integers $a \geq 0, c \geq 0, j \geq 0, b>0$ and $d>0$, let $P_{j}=U_{a+b j} U_{c+d j}$, $Q_{j}=U_{a+b j} V_{c+d j}$ and $\bar{R}_{j}=V_{a+b j} V_{c+d j}$. In [1] some formulae for the sums $\sum_{j=0}^{n} P_{j}, \sum_{j=0}^{n} Q_{j}, \sum_{j=0}^{n} R_{j}, \sum_{j=0}^{n}(-1)^{j} P_{j}, \sum_{j=0}^{n}(-1)^{j} Q_{j}$ and $\sum_{j=0}^{n}(-1)^{j} R_{j}$ have been discovered in the special case when $b=d=2$ and $q= \pm 1$. Even in these restricted case they gave unification of earlier results by Čerin and by Čerin and Gianella for Fibonacci, Lucas, Pell and Pell-Lucas numbers (see [3] - [9]).

In [2] the author eliminated all restrictions from the article [1] on $b, d$ and $q$ (except that $q \neq 0$ ). Some other types of sums have also been studied like the improved alternating sums (when we multiply terms by increasing powers of a fixed complex number), the sums with binomial coefficients and sums in which we multiply terms by increasing natural numbers.

The goal in this paper is to extend these results to Horadam type generalized Fibonacci numbers. Even in this more general case these sums could be evaluated using the sum of a geometric series.

Received May 6, 2008; accepted May 26, 2009.

2000 Mathematics Subject Classification: Primary 11B39, 11Y55, 05A19.

Key words and phrases: Horadam generalized Fibonacci numbers, Lucas numbers, sums of products. 


\section{Sums of products of two Horadam numbers}

We first want to find the formula for the sum

$$
\Psi_{1}=\sum_{j=0}^{n} w_{a+b j}\left(a_{0}, b_{0} ; p, q\right) w_{c+d j}\left(c_{0}, d_{0} ; p, q\right)
$$

when $a_{0}, b_{0}, c_{0}, d_{0}, p$ and $q \neq 0$ are complex numbers and $n \geq 0, a \geq 0, c \geq 0, b>0$ and $d>0$ are integers.

Let $\alpha$ and $\beta$ be the roots of $x^{2}-p x+q=0$. Then $\alpha=\frac{p+\Delta}{2}$ and $\beta=\frac{p-\Delta}{2}$, where $\Delta=\sqrt{p^{2}-4 q}$. Moreover, $\alpha-\beta=\Delta, \alpha+\beta=p, \alpha \beta=q$ and the Binet forms of $w_{n}, U_{n}$ and $V_{n}$ are

$$
w_{n}=\frac{\left(b_{0}-a_{0} \beta\right) \alpha^{n}+\left(a_{0} \alpha-b_{0}\right) \beta^{n}}{\alpha-\beta}, \quad U_{n}=\frac{\alpha^{n}-\beta^{n}}{\alpha-\beta}, \quad V_{n}=\alpha^{n}+\beta^{n},
$$

if $\alpha \neq \beta$, and

$$
w_{n}=\alpha^{n-1}\left(a_{0} \alpha+n\left(b_{0}-a_{0} \alpha\right)\right), \quad U_{n}=n \alpha^{n-1}, \quad V_{n}=2 \alpha^{n},
$$

if $\alpha=\beta$.

Let $A_{1}=b_{0}-a_{0} \alpha, \quad A_{2}=d_{0}-c_{0} \alpha, \quad B_{1}=a_{0} \beta-b_{0}, \quad B_{2}=c_{0} \beta-d_{0} . \quad$ Let $E=\alpha^{b+d}, F=\alpha^{b} \beta^{d}, G=\alpha^{d} \beta^{b}$ and $H=\beta^{b+d}$. Let $e=\alpha^{a+c} B_{1} B_{2}, f=\alpha^{a} \beta^{c} A_{2} B_{1}$, $g=\alpha^{c} \beta^{a} A_{1} B_{2}$ and $h=\beta^{a+c} A_{1} A_{2}$. When $E \neq 1$, for any integer $n \geq 0$, let $E_{n}=\frac{E^{n+1}-1}{E-1}$. We similarly define $F_{n}, G_{n}$ and $H_{n}$. On the other hand, when $\alpha^{b} \neq \beta^{b}$, for any integer $n \geq 0$, let $b_{n}=\frac{\alpha^{b(n+1)}-\beta^{b(n+1)}}{\alpha^{b n}\left(\alpha^{b}-\beta^{b}\right)}$ and $b_{n}^{*}=\frac{\alpha^{b(n+1)}-\beta^{b(n+1)}}{\beta^{b n}\left(\alpha^{b}-\beta^{b}\right)}$. We similarly define $d_{n}$ and $d_{n}^{*}$. For any integer $n \geq 0$, let $\lambda_{n}=n+1$. Let $T=\alpha^{a+c-2}$.

Let $C_{1}=A_{1} a+a_{0} \alpha, C_{2}=A_{2} c+c_{0} \alpha, K_{1}=b d A_{1} A_{2}, K_{3}=C_{1} C_{2}$ and $K_{2}=b A_{1} C_{2}+d A_{2} C_{1}$. Let $K_{4}=K_{1}+K_{2}+K_{3}$.

Let $K, M, N$ and $P$ be $n(2 n+1) K_{1}+3 n K_{2}+6 K_{3}$,

$$
\begin{gathered}
n^{2} E^{n+3}-\left(2 n^{2}+2 n-1\right) E^{n+2}+(n+1)^{2} E^{n+1}-E(E+1), \\
n E^{n+3}-(2 n+1) E^{n+2}+(n+1) E^{n+1}+E(E-1), \\
E^{n+3}-2 E^{n+2}+E^{n+1}-(E-1)^{2} .
\end{gathered}
$$

Theorem 1. (a) When $\Delta=0$ and $E=1$, then $\Psi_{1}=\frac{(n+1) K T}{6}$.

(b) When $\Delta=0$ and $E \neq 1$, then $\Psi_{1}=\frac{T\left[K_{1} M+K_{2} N+K_{3} P\right]}{(E-1)^{3}}$.

Proof. (a) Recall that when $\Delta=0$, then

$$
w_{a+b j}\left(a_{0}, b_{0} ; p, q\right)=\alpha^{a-1+b j}\left[b A_{1} j+C_{1}\right]
$$


and

$$
w_{c+d j}\left(c_{0}, b_{0} ; p, q\right)=\alpha^{c-1+d j}\left[d A_{2} j+C_{2}\right] .
$$

Since, $E=\alpha^{b+d}=1$, we see that the product

$$
w_{a+b j}\left(a_{0}, b_{0} ; p, q\right) w_{c+d j}\left(c_{0}, d_{0} ; p, q\right)
$$

is equal to

$$
T\left[K_{1} j^{2}+K_{2} j+K_{3}\right] .
$$

From $\sum_{j=0}^{n} 1=n+1, \sum_{j=0}^{n} j=\frac{n(n+1)}{2}$, and $\sum_{j=0}^{n} j^{2}=\frac{n(n+1)(2 n+1)}{6}$, it follows that $\Psi_{1}$ has the above value.

(b) Since $\Delta=0$, the product

$$
w_{a+b j}\left(a_{0}, b_{0} ; p, q\right) w_{c+d j}\left(c_{0}, d_{0} ; p, q\right)
$$

is equal to

$$
T E^{j}\left[K_{1} j^{2}+K_{2} j+K_{3}\right] .
$$

From $\sum_{j=0}^{n} E^{j}=\frac{P}{(E-1)^{3}}, \sum_{j=0}^{n} j E^{j}=\frac{N}{(E-1)^{3}}$, and $\sum_{j=0}^{n} j^{2} E^{j}=\frac{M}{(E-1)^{3}}$, it follows that $\Psi_{1}$ has the above value.

The following theorem covers for the sum $\Psi_{1}$ the cases when $\Delta \neq 0$. It uses Table 1 that should be read as follows. The symbols $\boldsymbol{\square}$ and $\square$ in column $E$ mean $E \neq 1$ and $E=1$. In column $b$ they mean $\alpha^{b} \neq \beta^{b}$ and $\alpha^{b}=\beta^{b}$. In columns $F$, $G, H$ and $d$ they have analogous meanings. The third subcase should be read as follows: When $(\Delta \neq 0), E=1$ and $\alpha^{b}=\beta^{b}$, then $G=1$ and $H=F$ and for $F \neq 1$ the product $\Delta^{2} \Psi_{1}$ is equal to $\lambda_{n}(e+g)+F_{n}(f+h)$.

Theorem 2. When $\Delta \neq 0$, then Table 1 gives the value of $\Delta^{2} \Psi_{1}$. In all other cases the product $\Delta^{2} \Psi_{1}$ is equal to $\lambda_{n}(e+f+g+h)$.

Proof of row 1 . When $\Delta \neq 0$, we have

$$
w_{a+b j}\left(a_{0}, b_{0} ; p, q\right)=-\frac{1}{\Delta}\left[\alpha^{a} B_{1}\left(\alpha^{b}\right)^{j}+\beta^{a} A_{1}\left(\beta^{b}\right)^{j}\right]
$$

and

$$
w_{c+d j}\left(c_{0}, d_{0} ; p, q\right)=-\frac{1}{\Delta}\left[\alpha^{c} B_{2}\left(\alpha^{d}\right)^{j}+\beta^{c} A_{2}\left(\beta^{d}\right)^{j}\right] .
$$

Hence, the product $w_{a+b j}\left(a_{0}, b_{0} ; p, q\right) w_{c+d j}\left(c_{0}, d_{0} ; p, q\right)$ is equal to

$$
\frac{e E^{j}}{\Delta^{2}}+\frac{f F^{j}}{\Delta^{2}}+\frac{g G^{j}}{\Delta^{2}}+\frac{h H^{j}}{\Delta^{2}} .
$$

From $\sum_{j=0}^{n} E^{j}=E_{n}$, we get $\Delta^{2} \Psi_{1}=e E_{n}+f F_{n}+g G_{n}+h H_{n}$. 


\begin{tabular}{|c|c|c|c|c|c|c|c|}
\hline & $E$ & $\bar{F}$ & $\bar{G}$ & $\bar{H}$ & $b$ & $d$ & $\Delta^{2} \Psi_{1}$ \\
\hline 1 & $\mathbf{\square}$ & $\mathbf{\square}$ & $\mathbf{\square}$ & $\mathbf{\square}$ & & & $E_{n} e+F_{n} f+G_{n} g+H_{n} h$ \\
\hline 2 & $\square$ & 口 & & [ & 口 & & $\lambda_{n} e+F_{n} f+b_{n} g+H_{n} h$ \\
\hline 3 & $\square$ & [ & $\nabla$ & $F$ & $\square$ & & $\lambda_{n}(e+g)+F_{n}(f+h)$ \\
\hline 4 & $\square$ & & $\mathbf{\square}$ & $\mathbf{\square}$ & & [ & $\lambda_{n} e+d_{n} f+G_{n} g+H_{n} h$ \\
\hline 5 & $\square$ & $\nabla$ & [ & $G$ & & $\square$ & $\lambda_{n}(e+f)+G_{n}(g+h)$ \\
\hline 6 & $\square$ & $\square$ & & & & 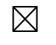 & $($ see 5$)$ \\
\hline 7 & $\square$ & & $\square$ & & 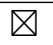 & & (see 3) \\
\hline 8 & $\square$ & & $\square$ & & & [ & $\lambda_{n}(e+g)+d_{n}(f+h)$ \\
\hline 9 & $\square$ & & & $\square$ & & $\mathbf{\square}$ & $\lambda_{n}(e+h)+d_{n} f+d_{n}^{*} g$ \\
\hline 10 & $\mathbf{\square}$ & $\square$ & $\mathbf{\square}$ & & $\mathbf{\square}$ & & $E_{n} e+\lambda_{n} f+G_{n} g+b_{n} h$ \\
\hline 11 & $\mathbf{\square}$ & $\square$ & $E$ & $\nabla$ & $\square$ & & $E_{n}(e+g)+\lambda_{n}(f+h)$ \\
\hline 12 & & $\square$ & $\mathbf{\square}$ & $\mathbf{\square}$ & & $\mathbf{\square}$ & $d_{n}^{*} e+\lambda_{n} f+G_{n} g+H_{n} h$ \\
\hline 13 & $\nabla$ & $\square$ & [ & $G$ & & $\square$ & $\lambda_{n}(e+f)+G_{n}(g+h)$ \\
\hline 14 & & $\square$ & $\square$ & & & $\mathbf{\square}$ & $d_{n}^{*} e+\lambda_{n}(f+g)+d_{n} h$ \\
\hline 15 & & $\square$ & & $\square$ & $\nabla$ & & $($ see 11$)$ \\
\hline 16 & & $\square$ & & $\square$ & & [ & $d_{n}^{*}(e+g)+\lambda_{n}(f+h)$ \\
\hline 17 & & $\mathbf{\square}$ & $\square$ & $\mathbf{\square}$ & [ & & $b_{n}^{*} e+F_{n} f+\lambda_{n} g+H_{n} h$ \\
\hline 18 & $\nabla$ & $\mathbf{\square}$ & $\square$ & $F$ & $\square$ & & $\lambda_{n}(e+g)+F_{n}(f+h)$ \\
\hline 19 & $\mathbf{\square}$ & $\mathbf{\square}$ & $\square$ & & & $\mathbf{\square}$ & $E_{n} e+F_{n} f+\lambda_{n} g+d_{n} h$ \\
\hline 20 & $\mathbf{\square}$ & $E$ & $\square$ & $\nabla$ & & $\square$ & $E_{n}(e+f)+\lambda_{n}(g+h)$ \\
\hline 21 & & & $\square$ & $\square$ & $\mathbf{\square}$ & $\bar{\nabla}$ & $b_{n}^{*}(e+f)+\lambda_{n}(g+h)$ \\
\hline 22 & [ & & [ & $\square$ & 口 & & $E_{n} e+b_{n}^{*} f+G_{n} g+\lambda_{n} h$ \\
\hline 23 & $\mathbf{\square}$ & 凶 & $E$ & $\square$ & $\square$ & & $E_{n}(e+g)+\lambda_{n}(f+h)$ \\
\hline 24 & [ & $\mathbf{\square}$ & & $\square$ & & [ & $E_{n} e+F_{n} f+d_{n}^{*} g+\lambda_{n} h$ \\
\hline 25 & $\square$ & $E$ & $\nabla$ & $\square$ & & $\square$ & $E_{n}(e+f)+\lambda_{n}(g+h)$ \\
\hline
\end{tabular}

Table 1: The product $\Delta^{2} \Psi_{1}$ when $\Delta \neq 0$. 
Proof of row 2. When $\Delta \neq 0$ and $E=\alpha^{b+d}=1$, we get

$$
w_{a+b j}\left(a_{0}, b_{0} ; p, q\right) w_{c+d j}\left(c_{0}, d_{0} ; p, q\right)=\frac{e}{\Delta^{2}}+\frac{f F^{j}}{\Delta^{2}}+\frac{g}{\Delta^{2}}\left(\frac{\beta^{b}}{\alpha^{b}}\right)^{j}+\frac{h H^{j}}{\Delta^{2}} .
$$

From $\sum_{j=0}^{n} 1=\lambda_{n}, \sum_{j=0}^{n} F^{j}=F_{n}$ and $\sum_{j=0}^{n}\left(\frac{\beta^{b}}{\alpha^{b}}\right)^{j}=b_{n}\left(\right.$ for $\alpha^{b} \neq \beta^{b}$ ), it follows that $\Delta^{2} \Psi_{1}=e \lambda_{n}+f F_{n}+g b_{n}+h H_{n}$.

Proof of row 3. When $\Delta \neq 0, E=\alpha^{b+d}=1$ and $\alpha^{b}=\beta^{b}$, then

$$
G=\beta^{b} \alpha^{d}=\alpha^{b} \alpha^{d}=E=1
$$

and $H=\beta^{b} \beta^{d}=\alpha^{b} \beta^{d}=F$. Hence,

$$
w_{a+b j}\left(a_{0}, b_{0} ; p, q\right) w_{c+d j}\left(c_{0}, d_{0} ; p, q\right)=\frac{e+g}{\Delta^{2}}+\frac{(f+h) F^{j}}{\Delta^{2}} .
$$

From $\sum_{j=0}^{n} 1=\lambda_{n}$ and $\sum_{j=0}^{n} F^{j}=F_{n}$ (for $F \neq 1$, of course), it follows that the product $\Delta^{2} \Psi_{1}$ is equal to $(e+g) \lambda_{n}+(f+h) F_{n}$.

The missing case in the Table 1 after the third row is clearly when $E=1$, $\alpha^{b}=\beta^{b}$ and $F=1$. The above product is

$$
w_{a+b j}\left(a_{0}, b_{0} ; p, q\right) w_{c+d j}\left(c_{0}, d_{0} ; p, q\right)=\frac{e+f+g+h}{\Delta^{2}},
$$

so that $\Delta^{2} \Psi_{1}=\lambda_{n}(e+f+g+h)$. The selection $p=0, q=-1, b=2$ and $d=2$ shows that this case can actually happen.

Notice that $\alpha^{n}=\frac{V_{n}+\Delta U_{n}}{2}$ and $\beta^{n}=\frac{V_{n}-\Delta U_{n}}{2}$ for $\Delta \neq 0$ and $\alpha^{n}=\beta^{n}=$ $\frac{\widetilde{U}_{n+1}}{n+1}=\frac{\widetilde{V}_{n}}{2}$ for $\Delta=0$. Hence, it is clear that each of the above expressions for the sum $\Psi_{1}$ could be transformed into an expression in Lucas numbers $U_{n}$ and $V_{n}$ (or $\widetilde{U}_{n}$ and $\left.\widetilde{V}_{n}\right)$. In most cases these formulae are more complicated then the ones given above. This applies also to other sums that we consider in this paper.

\section{Sum with binomial coefficients}

In this section we consider the sum

$$
\Psi_{2}=\sum_{j=0}^{n}\left(\begin{array}{l}
n \\
j
\end{array}\right) w_{a+b j}\left(a_{0}, b_{0} ; p, q\right) w_{c+d j}\left(c_{0}, d_{0} ; p, q\right),
$$

when $a_{0}, b_{0}, c_{0}, d_{0}, p$ and $q \neq 0$ are complex numbers and $n \geq 0, a \geq 0, c \geq 0, b>0$ and $d>0$ are integers.

Let $V$ and $U$ be $n E\left[(n E+1) K_{1}+(E+1) K_{2}\right]+(E+1)^{2} K_{3}$ and $E K_{4}+K_{3}$. 
Theorem 3. (a) When $\Delta=0$, then

$$
\Psi_{2}= \begin{cases}T K_{3}, & \text { if } n=0 \\ T U, & \text { if } n=1, \\ T(E+1)^{n-2} V, & \text { if } n \geq 2,\end{cases}
$$

(b) When $\Delta \neq 0$, then

$$
\Psi_{2}=\frac{(E+1)^{n} e+(F+1)^{n} f+(G+1)^{n} g+(H+1)^{n} h}{\Delta^{2}} .
$$

Proof. (b) Since

$$
\left(\begin{array}{c}
n \\
j
\end{array}\right) w_{a+b j}\left(a_{0}, b_{0} ; p, q\right) w_{c+d j}\left(c_{0}, d_{0} ; p, q\right)=\left(\begin{array}{c}
n \\
j
\end{array}\right) \frac{e E^{j}+f F^{j}+g G^{j}+h H^{j}}{\Delta^{2}}
$$

from $\sum_{j=0}^{n}\left(\begin{array}{l}n \\ j\end{array}\right) E^{j}=(E+1)^{n}$, it follows that $\Psi_{2}$ indeed has the above value.

\section{The improved alternating sums, I}

In this section we consider the sums obtained from the sums $\Psi_{1}$ and $\Psi_{2}$ by multiplication of their terms with the powers of a fixed complex number $k$. When $k=-1$ we obtain the familiar alternating sums. More precisely, we study the sums

$$
\begin{gathered}
\Psi_{3}=\sum_{j=0}^{n} k^{j} w_{a+b j}\left(a_{0}, b_{0} ; p, q\right) w_{c+d j}\left(c_{0}, d_{0} ; p, q\right), \\
\Psi_{4}=\sum_{j=0}^{n} k^{j}\left(\begin{array}{c}
n \\
j
\end{array}\right) w_{a+b j}\left(a_{0}, b_{0} ; p, q\right) w_{c+d j}\left(c_{0}, d_{0} ; p, q\right),
\end{gathered}
$$

when $a_{0}, b_{0}, c_{0}, d_{0}, p$ and $q \neq 0$ are complex numbers and $n \geq 0, a \geq 0, c \geq 0, b>0$ and $d>0$ are integers.

Let $E=k \alpha^{b+d}, F=k \alpha^{b} \beta^{d}, G=k \alpha^{d} \beta^{b}$ and $H=k \beta^{b+d}$. When $E \neq 1$, for any integer $n \geq 0$, let $E_{n}=\frac{E^{n+1}-1}{E-1}$. We similarly define $F_{n}, G_{n}$ and $H_{n}$.

In this section we can assume that $k \neq 1$ and $k \neq 0$ because the case when $k=1$ was treated earlier while for $k=0$ all sums are equal to zero.

With this new meaning of the symbols $E, F, G$ and $H$ we have the following result.

Theorem 4. (a) The values given in Theorems 1 and 2 express the sum $\Psi_{3}$. In particular, when $\Delta \neq 0$, then the Table 1 gives the values of $\Delta^{2} \Psi_{3}$. In all other cases the product $\Delta^{2} \Psi_{3}$ is equal to $\lambda_{n}(e+f+g+h)$.

(b) The values given in Theorem 3 for the sums $\Psi_{2}$ express also the sum $\Psi_{4}$. 
Proof. (b) Since

$$
k^{j}\left(\begin{array}{c}
n \\
j
\end{array}\right) w_{a+b j}\left(a_{0}, b_{0} ; p, q\right) w_{c+d j}\left(c_{0}, d_{0} ; p, q\right)=\left(\begin{array}{c}
n \\
j
\end{array}\right) \frac{e E^{j}+f F^{j}+g G^{j}+h H^{j}}{\Delta^{2}}
$$

from $\sum_{j=0}^{n}\left(\begin{array}{c}n \\ j\end{array}\right) E^{j}=(E+1)^{n}$, it follows that $\Psi_{4}$ indeed has the same expression as the sum $\Psi_{2}$.

\section{Terms multiplied by natural numbers}

In this section we study the sums

$$
\begin{gathered}
\Psi_{5}=\sum_{j=0}^{n}(j+1) w_{a+b j}\left(a_{0}, b_{0} ; p, q\right) w_{c+d j}\left(c_{0}, d_{0} ; p, q\right), \\
\Psi_{6}=\sum_{j=0}^{n}(j+1)\left(\begin{array}{c}
n \\
j
\end{array}\right) w_{a+b j}\left(a_{0}, b_{0} ; p, q\right) w_{c+d j}\left(c_{0}, d_{0} ; p, q\right),
\end{gathered}
$$

when $a_{0}, b_{0}, c_{0}, d_{0}, p$ and $q \neq 0$ are complex numbers and $n \geq 0, a \geq 0, c \geq 0, b>0$ and $d>0$ are integers.

Let $E=\alpha^{b+d}, F=\alpha^{b} \beta^{d}, G=\alpha^{d} \beta^{b}, H=\beta^{b+d}$. Let $e=\alpha^{a+c} B_{1} B_{2}$, $f=\alpha^{a} \beta^{c} A_{2} B_{1}, g=\alpha^{c} \beta^{a} A_{1} B_{2}, h=\beta^{a+c} A_{1} A_{2}$. When $E \neq 1$, for any integer $n \geq 0$, let $E_{n}=\frac{(n+1) E^{n+2}-(n+2) E^{n+1}+1}{(E-1)^{2}}$. We similarly define $F_{n}, G_{n}$ and $H_{n}$. On the other hand, when $\alpha^{b} \neq \beta^{b}$, for any integer $n \geq 0$, let

$$
b_{n}=\frac{\alpha^{b(n+2)}+(n+1) \beta^{b(n+2)}-(n+2) \alpha^{b} \beta^{n+1}}{\alpha^{b n}\left(\alpha^{b}-\beta^{b}\right)^{2}}
$$

and

$$
b_{n}^{*}=\frac{\beta^{b(n+2)}+(n+1) \alpha^{b(n+2)}-(n+2) \beta^{b} \alpha^{n+1}}{\beta^{b n}\left(\alpha^{b}-\beta^{b}\right)^{2}} .
$$

We similarly define $d_{n}$ and $d_{n}^{*}$. For any integer $n \geq 0$, let $\lambda_{n}=\frac{(n+1)(n+2)}{2}$.

Let $M$ and $N$ denote $n E^{n+3}\left[n(n+1) E-\left(3 n^{2}+6 n-1\right)\right]+(n+2)$ $E^{n+1}\left[\left(3 n^{2}+3 n-2\right) E-(n+1)^{2}\right]+2 E(2 E+1)$ and $(E-1)\left[n(n+1) E^{n+3}-2 n(n+2) E^{n+2}+(n+2)(n+1) E^{n+1}-2 E\right]$.

Theorem 5. (a) When $\Delta=0$ and $E=1$, then the sum $\Psi_{5}$ is equal to

$$
\frac{\lambda_{n} T\left[n(3 n+1) K_{1}+4 n K_{2}+6 K_{3}\right]}{6} .
$$

(b) When $\Delta=0$ and $E \neq 1$, then the sum $\Psi_{5}$ is equal to

$$
\frac{T}{(E-1)^{4}}\left[K_{1} M+K_{2} N+K_{3}(E-1)^{4} E_{n}\right] \text {. }
$$


Proof. (b) Since $\Delta=0$, we have

$$
\begin{aligned}
(j+1) w_{a+b j} & \left(a_{0}, b_{0} ; p, q\right) w_{c+d j}\left(c_{0}, d_{0} ; p, q\right) \\
& =(j+1)\left(\alpha^{a+b j-1}\left[b A_{1} j+C_{1}\right]\right)\left(\alpha^{c+d j-1}\left[d A_{2} j+C_{2}\right]\right) \\
& =(j+1) T E^{j}\left[K_{1} j^{2}+K_{2} j+K_{3}\right] .
\end{aligned}
$$

From $\sum_{j=0}^{n}(j+1) E^{j}=E_{n}, \sum_{j=0}^{n} j(j+1) E^{j}=\frac{N}{(E-1)^{4}}$, and

$$
\sum_{j=0}^{n} j^{2}(j+1) E^{j}=\frac{M}{(E-1)^{4}}
$$

it follows that $\Psi_{5}$ has the above value.

Theorem 6. When $\Delta \neq 0$, then the Table 1 gives the values of $\Delta^{2} \Psi_{5}$. In all other cases the product $\Delta^{2} \Psi_{5}$ is equal to $\lambda_{n}(e+f+g+h)$.

Proof of row 1 in Table 1 for $\Psi_{5}$. When $\Delta \neq 0$, we have

$(j+1) w_{a+b j}\left(a_{0}, b_{0} ; p, q\right) w_{c+d j}\left(c_{0}, d_{0} ; p, q\right)$

$$
\begin{aligned}
& =(j+1)\left(-\frac{1}{\Delta}\left[B_{1} \alpha^{a+b j}+A_{1} \beta^{a+b j}\right]\right)\left(-\frac{1}{\Delta}\left[B_{2} \alpha^{c+d j}+A_{2} \beta^{c+d j}\right]\right) \\
& =(j+1)\left(\frac{e E^{j}}{\Delta^{2}}+\frac{f F^{j}}{\Delta^{2}}+\frac{g G^{j}}{\Delta^{2}}+\frac{h H^{j}}{\Delta^{2}}\right) .
\end{aligned}
$$

From $\sum_{j=0}^{n}(j+1) E^{j}=E_{n}$, we get $\Delta^{2} \Psi_{5}=e E_{n}+f F_{n}+g G_{n}+h H_{n}$.

For any integer $n \geq 0$, let $E_{n}^{*}=(n+1) E+1, E_{n}^{* *}=E_{n}^{*}(E+1)^{n-1}$. We define $F_{n}^{*}, G_{n}^{*}, H_{n}^{*}, F_{n}^{* *}, G_{n}^{* *}$ and $H_{n}^{* *}$ similarly. Let

$$
M=n E(E+1)^{n-3}\left(E_{2 n-2}^{*}+E_{n}^{*} E_{n-1}^{*}\right), \quad N=n E(E+1)^{n-2}\left(E_{n}^{*}+1\right) .
$$

Theorem 7. (a) When $\Delta=0$, then

$$
\Psi_{6}= \begin{cases}T K_{3}, & \text { if } n=0, \\ T\left[2 E K_{4}+K_{3}\right], & \text { if } n=1, \\ T\left[3 E^{2}\left(K_{4}+K_{2}+3 K_{1}\right)+4 E K_{4}+K_{3}\right], & \text { if } n=2, \\ T\left[M K_{1}+N K_{2}+E_{n}^{* *} K_{3}\right], & \text { if } n \geq 3 .\end{cases}
$$

(b) When $\Delta \neq 0$, then $\Delta^{2} \Psi_{6}=E_{n}^{* *} e+F_{n}^{* *} f+G_{n}^{* *} g+H_{n}^{* *} h$.

Proof. (b) Since

$$
\begin{aligned}
(j+1)\left(\begin{array}{c}
n \\
j
\end{array}\right) w_{a+b j} & \left(a_{0}, b_{0} ; p, q\right) w_{c+d j}\left(c_{0}, d_{0} ; p, q\right) \\
& =\frac{1}{\Delta^{2}}(j+1)\left(\begin{array}{c}
n \\
j
\end{array}\right)\left(e E^{j}+f F^{j}+g G^{j}+h H^{j}\right),
\end{aligned}
$$


from $\sum_{j=0}^{n}(j+1)\left(\begin{array}{l}n \\ j\end{array}\right) E^{j}=E_{n}^{* *}$, it follows that $\Delta^{2} \Psi_{6}$ indeed has the above value.

\section{The improved alternating sums, II}

In this section we study the following sums obtained by multiplying the terms of the sums $\Psi_{5}$ and $\Psi_{6}$ with the powers of the fixed complex number $k$. Of course, for $k=1$, we get the sums $\Psi_{5}$ and $\Psi_{6}$ from the sums $\Psi_{7}$ and $\Psi_{8}$.

$$
\begin{gathered}
\Psi_{7}=\sum_{j=0}^{n} k^{j}(j+1) w_{a+b j}\left(a_{0}, b_{0} ; p, q\right) w_{c+d j}\left(c_{0}, d_{0} ; p, q\right), \\
\Psi_{8}=\sum_{j=0}^{n} k^{j}(j+1)\left(\begin{array}{c}
n \\
j
\end{array}\right) w_{a+b j}\left(a_{0}, b_{0} ; p, q\right) w_{c+d j}\left(c_{0}, d_{0} ; p, q\right),
\end{gathered}
$$

when $a_{0}, b_{0}, c_{0}, d_{0}, p$ and $q \neq 0$ are complex numbers and $n \geq 0, a \geq 0, c \geq 0, b>0$ and $d>0$ are integers.

Let $E=k \alpha^{b+d}, F=k \alpha^{b} \beta^{d}, G=k \alpha^{d} \beta^{b}$ and $H=k \beta^{b+d}$. When $E \neq 1$, for any integer $n \geq 0$, let $E_{n}=\frac{(n+1) E^{n+2}-(n+2) E^{n+1}+1}{(E-1)^{2}}$. We similarly define $F_{n}, G_{n}$ and $H_{n}$. On the other hand, when $\alpha^{b} \neq \beta^{b}$, for any integer $n \geq 0$, we define $b_{n}$ and $b_{n}^{*}$ as in the previous section. We similarly define $d_{n}$ and $d_{n}^{*}$. In this section $\lambda_{n}$ is again $\frac{(n+1)(n+2)}{2}$.

Theorem 8. The expressions for $\Psi_{5}$ in Theorems 5 and 6 describe also the sum $\Psi_{7}$ (with the new meaning of $E, F, G$ and $H$ ).

Theorem 9. The expressions for $\Psi_{6}$ in Theorem 7 describe also the sum $\Psi_{8}$ (with the new meaning of $E, F, G$ and $H$ ).

\section{References}

[1] H. Belbachir and F. Bencherif, Sums of products of generalized Fibonacci and Lucas numbers, Ars Combinatoria, (to appear).

[2] Z. Čerin, Sums of products of generalized Fibonacci and Lucas numbers, Demonstratio Mathematica, 42(2) (2009), 247-258.

[3] Z. Čerin, Properties of odd and even terms of the Fibonacci sequence, Demonstratio Mathematica, 39(1) (2006), 55-60.

[4] Z. Čerin, On sums of squares of odd and even terms of the Lucas sequence, Proccedings of the Eleventh International Conference on Fibonacci Numbers and their Applications, Congressus Numerantium, 194(2009), 103-107.

[5] Z. Čerin, Some alternating sums of Lucas numbers, Central European Journal of Mathematics 3(1) (2005), 1-13. 
[6] Z. Čerin, Alternating Sums of Fibonacci Products, Atti del Seminario Matematico e Fisico dell'Università di Modena e Reggio Emilia, 53(2005), 331-344.

[7] Z. Čerin and G. M. Gianella, On sums of squares of Pell-Lucas numbers, INTEGERS: Electronic Journal of Combinatorial Number Theory, 6(2006), A15.

[8] Z. Čerin and G. M. Gianella, Formulas for sums of squares and products of Pell numbers, Acc. Sc. Torino - Atti Sci. Fis., 140(2006), 113-122.

[9] Z. Črin and G. M. Gianella, On sums of Pell numbers, Acc. Sc. Torino - Atti Sci. Fis., 141(2007), 23-31.

[10] A. F. Horadam, Generating Functions for Powers of a Certain Generalized Sequence of Numbers, Duke. Math. J., 32(1965), 437-446.

[11] E. Lucas, Théorie des Fonctions Numériques Simplement Périodiques, American Journal of Mathematics 1(1878), 184-240.

[12] N. J. A. Sloane, On-Line Encyclopedia of Integer Sequences, http://www.research.att.com/ $\sim n j a s /$ sequences/. 